\title{
CMB Polarization with BICEP2 and Keck-Array
}

\section{Clement Pryke for the BICEP2 and Keck-Array Collaborations}

\author{
University of Minnesota Physics, 116 Church Street S.E., \\ Minneapolis, MN, 55455, USA
}

\begin{abstract}
BICEP2 is an evolution from the highly successful BICEP CMB polarization experiment. In turn Keck-Array is an array of BICEP2 like receivers to achieve an additional increase in sensitivity. All these experiments are located at the South Pole in Antarctica and target the CMB $B$-mode polarization signal which is predicted to exist in many simpler models of Inflation at angular scales of several degrees. The design and performance of BICEP2 and Keck-Array is described and some preliminary polarization maps are presented.
\end{abstract}

Keywords. CMB, Polarization, Antarctica

\section{Introduction}

The theory known as inflation posits that the Universe underwent a tremendous burst of hyper expansion (factor $\sim 10^{60}$ ) at a tiny fraction of a second after the initial singularity $\left(\sim 10^{-35} \mathrm{~s}\right)$. During this expansion quantum fluctuations of the metric of all kinds are "forced real" injecting — amongst other things — gravity waves into the fabric of space which then propagate through it until the present time. At $\approx 380,000$ years after the beginning the Universe made the transition from plasma to neutral gas (recombination) and the light we see today as the Cosmic Microwave Background (CMB) was released. If these Inflationary gravity waves were present at recombination then they will have produced quadrupolar variations in the intensity of the CMB photons as they were last scattered from the plasma electrons. When electrons are exposed to unpolarized light which has a quadrupolar anisotropy the re-scattered light is partially polarized. The inhomogeneities which give rise to the temperature anisotropy of the CMB drive flows of material which, through Dopler shifting, generate quadrupolar anisotropy and hence polarization. However the resulting polarization pattern is naturally aligned with its own gradient and, by analogy with electrodynamics, is known as $E$-mode. The gravity waves, on the other hand, respect no such rule and can produce patterns with a curl, or $B$-mode, component. A number of experiments are targeting this exciting, although perhaps elusive, signal. They are in essence remote sensing gravity wave experiments looking for the tell-tale signature of Inflation written on the last scattering surface.

The polarization of the CMB was first detected by the DASI experiment which was sited at South Pole (Kovac et al. 2009). DASI was followed at Pole by the twin experiments QUaD (Brown et al. 2009) and BICEP (Chiang et al. 2009) which together still hold the record for best sensitivity at smaller and larger angular scales respectively. BICEP has now been replaced by BICEP2 and QUaD with the Keck-Array. The science goal of all these experiments is the same - to push down to the sensitivity level required to detect the inflationary $B$-modes. The signal is conventionally described in terms of the tensor to scalar ratio $r$. From temperature anisotropy and other observations we know that $r \leqslant 0.2$. This paper will describe BICEP2 and Keck-Array and their preliminary results. 


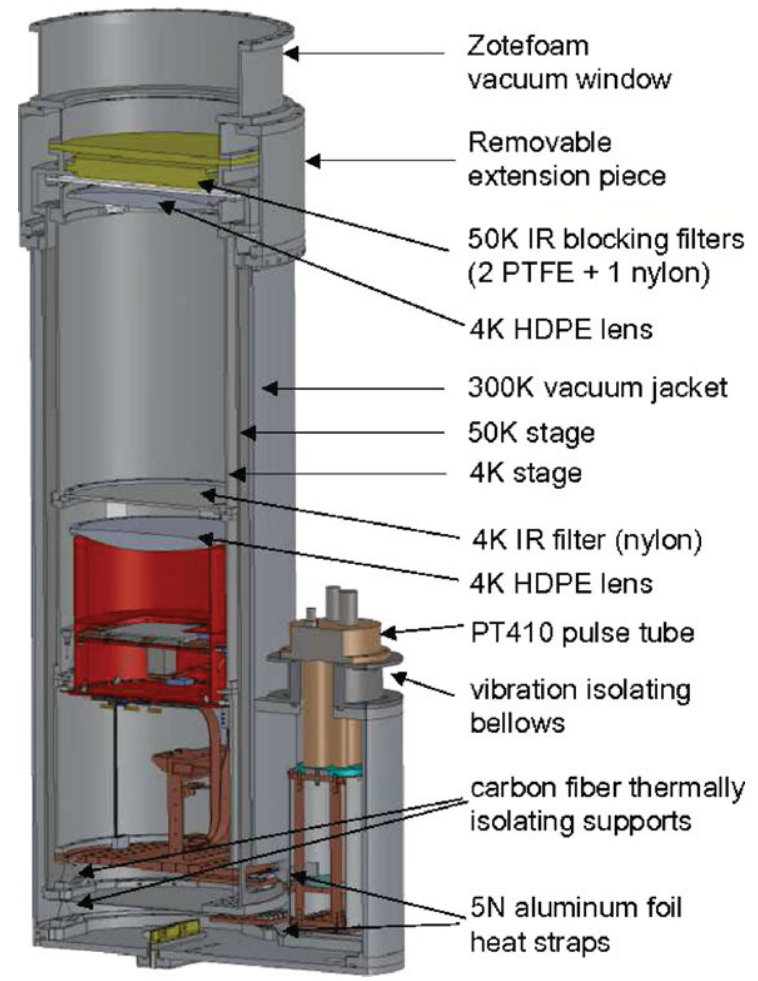

Figure 1. Cross section of a Keck-Array cryostat. (Reproduced from Sheehy et al. (2010).)

\section{The BICEP/Keck Strategy}

The BICEP/Keck strategy for the detection of inflationary $B$-modes is to go as deep as possible, as quickly as possible, at $150 \mathrm{GHz}$ in the "Southern Hole" region (centered at $\left.\mathrm{RA}=0^{h}, \mathrm{Dec}=-57^{\circ}\right)$. At this frequency the CMB is bright, but also maximally distinct from galactic foreground emission in such areas of high galactic latitude sky. The $\sim 600$ square degree patch size is selected to allow adequate separation of $E$ and $B$-modes at the angular scale where the cosmological signal is predicted to peak $(\ell \sim 80)$. The "Southern Hole" is also at high (and constant) elevation angle as viewed from the Pole which helps to reduce (and stabilize) possible ground contamination. The Sun is also a potential source of contamination but is always relatively far from the observing field and, of course, below the horizon for six months of the year.

The South Pole is an excellent site for these type of observations as is detailed elsewhere in these Proceedings. At $150 \mathrm{GHz}$ water vapor is highly opaque. Due to the high altitude and extreme cold the median precipitable water vapor is lower on the Antarctic plateau than at any other site $\dagger$. Importantly what moisture there is is well mixed in the atmosphere and thus presents a stable baseline to the observations.

BICEP2 and Keck-Array use near identical optics to BICEP1. The light enters through a $30 \mathrm{~cm}$ vacuum window, passes through IR blocking filters, and then through an all-cold telecentric refracting telescope (see Figure 1). This system delivers only modest angular resolution (0.6 deg FWHM at $150 \mathrm{GHz}$ ) but is highly stable, compact, and low cost. In addition, due to the small size, it can be fitted with a long absorptive fore-baffle which

$\dagger$ Some sites have lower PWV for a small fraction of the time but what matters for CMB observing is the "average" conditions. 


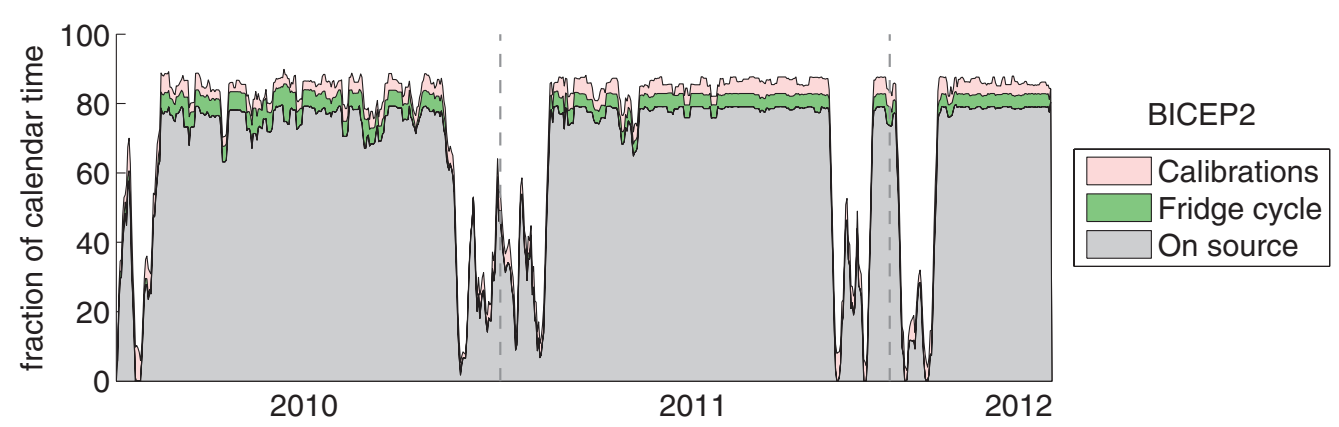

Figure 2. Observing efficiency of BICEP2 through 2.5 observing seasons. (Reproduced from Ogburn et al. (2012).)

results in extremely low sidelobe response. A further advantage of big beam telescopes is that the far field is only $\sim 100 \mathrm{~m}$ away enabling calibration sources to be placed on masts of modest height. The focal planes are cooled to $250 \mathrm{mK}$ using closed cycle helium refrigerators.

The basic operating principle of the telescopes is similar to many others. The mount scans in azimuth sweeping the pencil beams of the detectors across the sky while the detector readout signals are recorded. After a number of scans the mount steps in elevation and the process repeats forming a raster pattern. Each pixel contains bolometers sensitive to orthogonal linear polarizations. In offline analysis relative gain calibration is applied and the pair difference taken to measure polarization. The data is binned into maps with appropriate accounting for the angle of the pair as projected onto the sky. Atmospheric emission is unpolarized and so cancels in the differencing operation.

Working at Pole enforces a strong discipline on the experimental effort. For nine months of the year the station is "closed" — no planes can arrive or leave. Thus all equipment installation, maintenance and upgrades must be conducted during the brief summer season. Once the last plane departs the telescopes settle into relentless homogeneous observation watched over by their dedicated winter-over scientists.

\section{BICEP2}

While BICEP and QUaD had focal planes assembled from individually packaged NTD detectors (diced wafers) BICEP2 made the transition to monolithic tiles of detectors (intact wafers). These new generation focal planes consist of four tiles each containing 64 dual polarization pixels (i.e. 256 pixels and 512 total TES detectors). Instead of using feedhorns the beam forming is accomplished "on wafer" by making each pixel a phased array of planar crossed dipole antennas.

BICEP2 was deployed in late 2009 and has observed through the 2010, 2011 and 2012 Austral winters. Observing efficiency has been excellent as is seen in Figure 2. The per detector sensitivity is better than BICEP1 and the yield is also good - Figure 3 compares the sensitivity distributions - BICEP2 has more than ten times the mapping speed of BICEP1.

Although the analysis does not primarily rely on it, as a "by-product" it is possible to make maps of $E$-modes and $B$-modes. Figure 4 shows such maps made with six months of BICEP2 data - note the dramatic reduction in $B$-mode noise. Ground based polarization experiments benefit tremendously from performing an instantaneous pair difference of orthogonal detectors - low frequency noise from both atmosphere and instrument is almost completely common mode and the pair difference noise is empirically found to be 


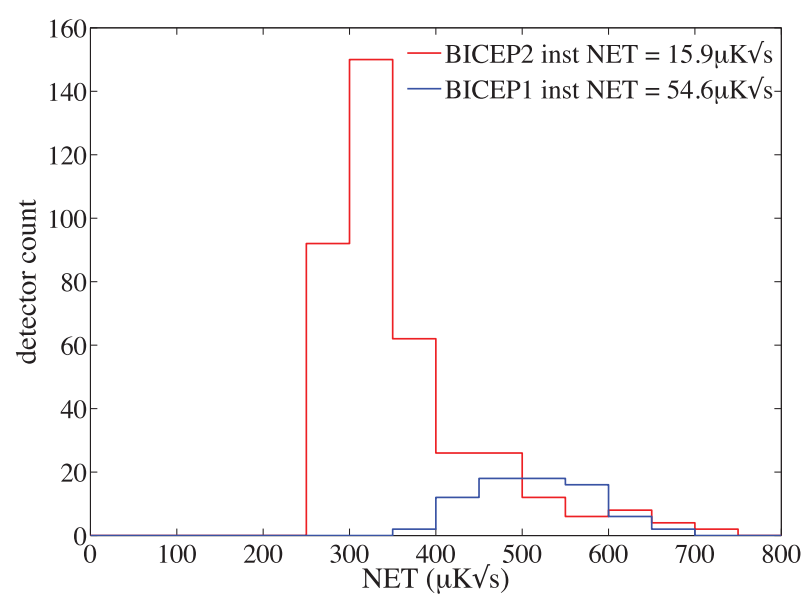

Figure 3. Distribution of sensitivity of BICEP2 detectors versus that of BICEP1. Note that not only does BICEP2 have many more pixels than BICEP1 but that each of them is also more sensitive. (Figure courtesy of Justus Brevik.)

almost white. (The alternative is fast modulation of the polarization sensitivity angle BICEP/Keck has not chosen to pursue this option.)

Pair differencing demands excellent spatial coincidence of the beams on the sky, and this requirement is especially demanding when the angular size of the beams is only a few times smaller than the effect under study. If there is a small offset in the $A / B$ beam centroids then this produces a leakage of the gradient of the much brighter temperature anisotropy into the polarization signal. However under rotation of the array by 180 degrees the leakage is inverted and hence cancels in the polarization map. The BICEP2 detectors exhibit substantial $A / B$ centroid offset but by designing the observation pattern appropriately the leakage is demonstrably suppressed by a large factor. This is visually demonstrated in Figure 4 where the $B$-mode map noise is reduced by a large factor versus BICEP1 - these maps effectively are a $B$-mode limit many times better than the published BICEP1 result (Chiang et al. 2009). Further suppression of centroid offset and other beam systematic effects is achieved in analysis using a technique dubbed "deprojection". A full analysis of the first two years of BICEP2 data is in an advanced state and an $r$-limit will be released very soon.

\section{Keck-Array}

The focal plane of BICEP2 is already packed with the maximum number of detectors which it will support with full efficiency and acceptable beam quality. Good though the sensitivity of BICEP2 is, pursuit of the gravity wave signal demands further increase in sensitivity. The only option then is to increase the numbers of receivers and this is exactly what Keck-Array does - deploys a set of five BICEP2 like receivers on the telescope mount originally built for DASI (and then re-used for QUaD). This was made possible by the transition from liquid helium cooling to pulse tube cooler technology, and in addition required a very compact cryostat design - see Figure 1. Note the extremely small (few centimeter) distance from the $300 \mathrm{~K}$ cryostat shell to the $4 \mathrm{~K}$ telescope assembly. While the pulse tube coolers use $\sim 10 \mathrm{~kW}$ each presenting a non negligible load on the station power plant, this is still logistically far preferable to the resources which would be necessary to provide vast amounts of liquid helium through each winter season. 


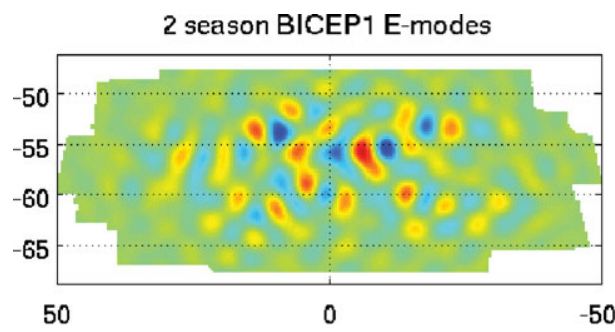

Half Season BICEP2 E-modes cut

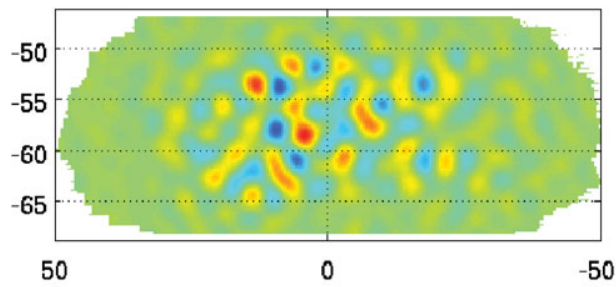

2 season BICEP1 B-modes

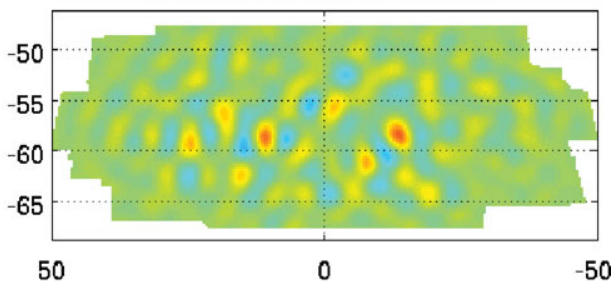

Half Season BICEP2 B-modes cut

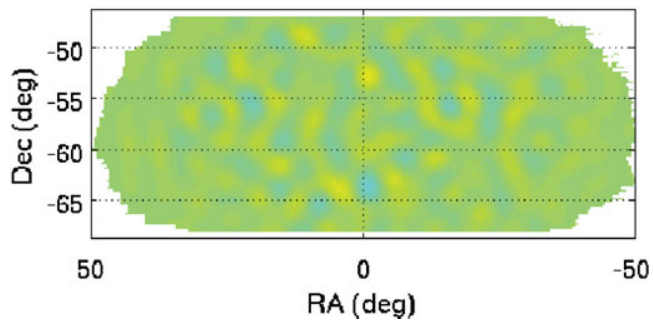

Figure 4. Maps of the CMB polarization $E$-mode and $B$-mode filtered to the angular scale range where the inflationary gravity wave signal is expected to be most prominent $(50<\ell<120)$. The upper maps are the published BICEP1 results which hold the current world record in terms of sensitivity $(r<0.72$, Chiang et al. (2010)). The lower maps are new preliminary BICEP2 results using half a season of data. The $E$-mode maps were already signal dominated for BICEP1 and so show little change going to BICEP2. However the BICEP2 $B$-mode maps show a large decrease in noise due to the much higher sensitivity of the BICEP2 instrument.

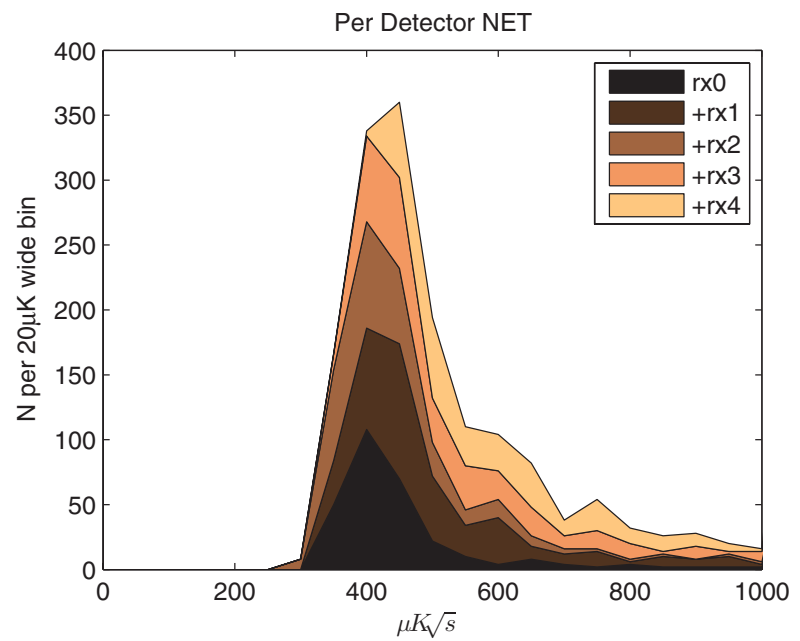

Figure 5. Histogram showing the sensitivity of Keck-Array detectors with a per receiver breakdown. Further improvements are planned - especially to the worst performing receiver. (Reproduced from Kernasovskiy et al. (2012).)

The sensitivity of the Keck-Array as measured during the 2012 Austral winter season is shown in Figure 5. One of the receivers badly underperforms and upgrades to this and others will be performed during the 2012/13 summer season.

The pulse tube coolers cease to function when inclined at angles $>45$ degrees from the vertical. (The line of sight rotation axis of the telescope mount precludes angling the cooler from the optical axis as is done for SPT.) Therefore a 45 degree flat mirror is 

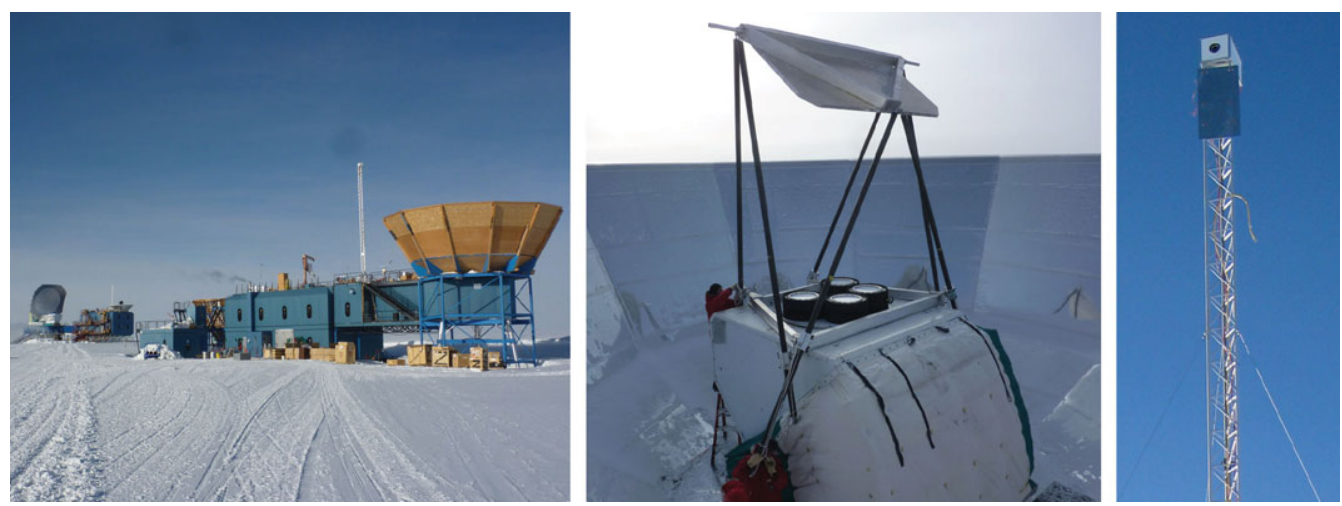

Figure 6. Calibration of Keck-Array. Left: Keck-Array is located in the bowl shaped shield in the foreground, while the calibration source is on the building in the background. Center: The flat mirror mounted on Keck-Array. Right: A calibration source transmits from its mast. (Reproduced from Vieregg et al. (2012).)
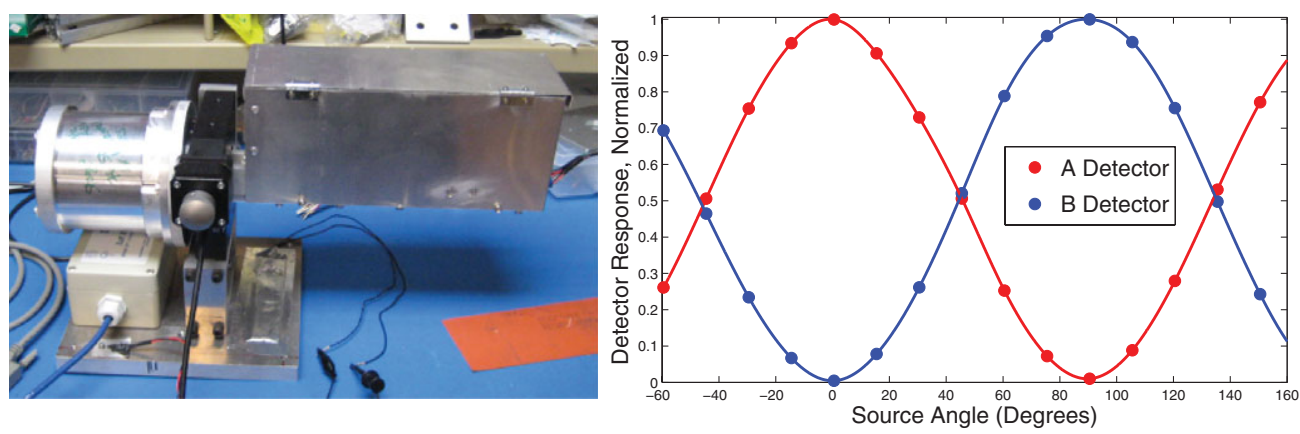

Figure 7. A rotating polarized calibration source and test results. Note the excellent polarization purity of the new antenna coupled detectors. (Reproduced from Vieregg et al.

(2012).)

required to redirect the beams parallel to the horizon to calibration sources mounted on an adjacent building. Because of the large bowl shaped shield within which the mount is located this mirror has to be extremely large and high above the telescopes. In addition there is a strong desire to be able to mount and unmount this calibration flat without the use of a crane. An elegant solution has been implemented using honeycomb panels and large carbon fiber rods - see Figure 6.

Figure 7 shows a rotating polarized source and test data. The source transmits near $100 \%$ polarized broadband radiation while the entire assembly is rotated. The stationary detectors record data which modulates sinusoidally as the source aligns and anti-aligns with the polarization sensitivity direction of each given detector. The polarization purity of the new antenna coupled detectors is excellent - when anti-aligned the signal is $<1 \%$ of that when aligned. (The older horn fed detectors had cross polar response of $\sim 3 \%$.)

Figure 8 shows test data taken with a chopped thermal (unpolarized) source. In this case the telescope scans out a raster pattern passing the beam of each detector across the source direction, thus producing a beam map. The illumination of the aperture plane is a truncated Gaussian leading to a far field beam pattern with an approximately Gaussian main lobe surrounded by ring sidelobes. The figure shows the beam of a single detector with an elliptical Gaussian fit and the residual. The first sidelobe is apparent and stacking over detectors multiple sidelobes are detected in excellent agreement with optical 

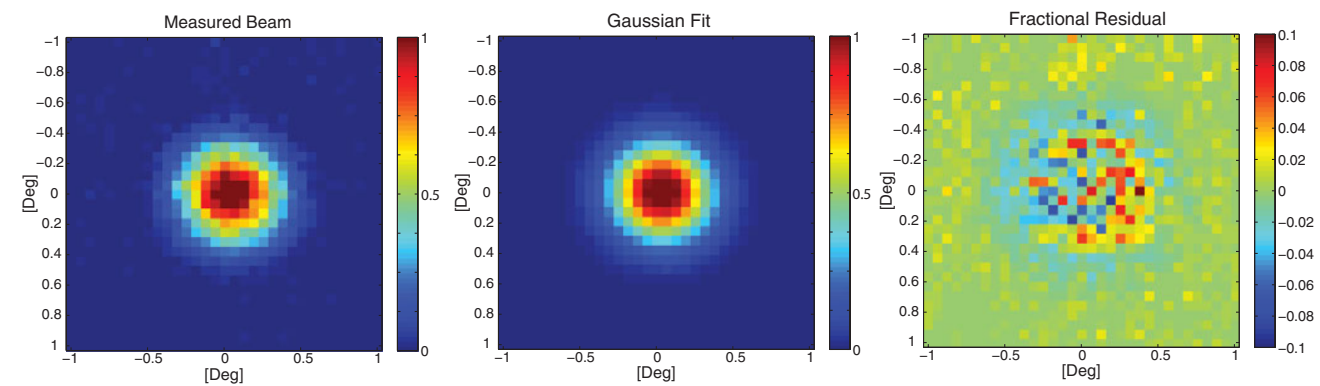

Figure 8. Left: far field beam pattern of a single Keck-Array detector as measured with an unpolarized thermal source. Center: the best fitting elliptical Gaussian. Right: the residual between data and model. (Reproduced from Vieregg et al. (2012).)

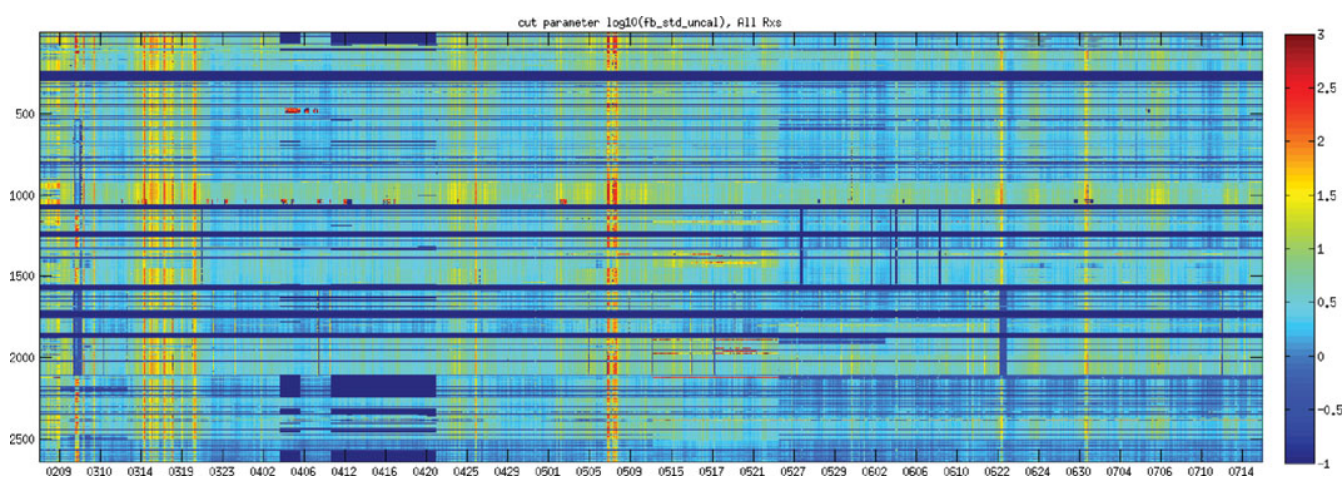

Figure 9. One of the Keck-Array cut parameters which monitors the raw data quality. The color scale shows the log of the standard deviation of the data as measured across each forward or backward "half-scan". Red/yellow vertical bars correspond to bad weather while horizontal dark blue bands are non functional pixels. The dark blue vertical bar around 0622 corresponds to a period when one of the five receivers was offline. Note that the horizontal scale is approaching six months.

modeling. Further beam mapping is planned although this data is mainly a cross check and is not explicitly needed for CMB analysis. The deprojection algorithm effectively fits for the beam parameters from the CMB data itself. The $A / B$ centroid offsets of the Keck-Array detectors are somewhat smaller than that of BICEP2 and further major improvements have been achieved in newer test detectors.

Keck-Array is an extremely complicated system with $\sim 2500$ channels of TES detectors being read out through several layers of SQUID's. There are many parameters to tune and much scope for optimization. During winter running the data is transferred back to the US daily via satellite $(\sim 25 \mathrm{~GB} /$ day $)$ and must be constantly monitored for quality assurance. To aid in this process, and as an integral part of the data reduction, sophisticated automatic data cutting machinery has been built. Figure 9 shows one of the lowest level cut parameters.

\section{Conclusion}

The BICEP2 and Keck-Array experiments have been described. As of the time of writing BICEP2 is completing its third and final season of observations. Meanwhile KeckArray is completing its first season with the full complement of five receivers. Four more seasons of observations are planned with further sensitivity upgrades and a switch to 
additional observing frequencies (90 and/or $220 \mathrm{GHz}$ ). Meanwhile a published result from BICEP2 is imminent. With the massive sensitivity which Keck-Array brings to bear detection of $B$-mode polarization is essentially guaranteed. Our projections indicate that the $B$-mode from galactic foreground in our field will be at a level equivalent to $r \sim 0.02$ with an uncertainty of perhaps factor two. If a signal is detected then the angular power spectrum will give hints as to whether the origin is galactic or cosmological. Ultimately however confirmation at other frequencies and by other experiments will be required.

\section{References}

J. Kovac, E. Leitch, C. Pryke, J. Carlstrom, \& W. Holzapfel 2001, Nature, 442, 772. astro$\mathrm{ph} / 0209478$

M. Brown et al. 2009, ApJ, 705, 978. arxiv/0906.1003

C. Chiang et al. 2010, ApJ, 711, 1123. arxiv/0906.1181

R. W. Ogburn et al. 2012, Proceedings of SPIE, 8452. arxiv/1208.0638

C. Sheehy et al. 2010, Proceedings of SPIE, 7741. arxiv/1104.5516

S. Kernasovskiy et al. 2012, Proceedings of SPIE, 8452. arxiv/1208.0857

A. Vieregg et al. 2012, Proceedings of SPIE, 8452. arxiv/1208.0844 